\title{
NGC 6397: The metallicity trend along the isochrone revisited ${ }^{\star}$
}

\author{
Rashi Jain ${ }^{1}$, Philippe Prugniel ${ }^{2}$, Lucimara Martins ${ }^{3,2}$, and Ariane Lançon ${ }^{1}$ \\ 1 Université de Strasbourg, CNRS, Observatoire Astronomique de Strasbourg, UMR7550, 67000 Strasbourg, France \\ e-mail: r.jain@unistra.fr \\ 2 Centre de Recherche Astrophysique de Lyon UMR5574, Univ Lyon, Univ Lyon1, ENS de Lyon, CNRS, 69230 Saint-Genis-Laval, \\ France \\ ${ }^{3}$ NAT- Universidade Cidade de São Paulo/Universidade Cruzeiro do Sul, Rua Galvão Bueno, 868, 01506-000 Sao Paulo, Brazil
}

Received 18 October 2019 / Accepted 17 December 2019

\begin{abstract}
Context. Recent work has used spectra of $~ 5000$ stars in NGC 6397 that were extracted from a MUSE mosaic to determine the atmospheric parameters for these stars by fitting the spectra against the Göttingen Spectral Library. A significant change in metallicity between the turn off and the red giant branch was found and was discussed as a possible manifestation of predicted effects of atomic diffusion. However, the small amplitude of the effect and inconsistency with earlier measurements call for more attention before this result is interpreted. Systematic effects due to the interpolation or to the synthetic spectra cannot be ruled out at this level of precision. Aims. We reanalyze the data with : the ELODIE and MILES reference libraries in order to assess the robustness of the result. These empirical libraries have a finer metallicity coverage down to approximately the cluster metalicity turn-off.

Methods. We used the ULySS full-spectrum fitting package, together with the library interpolators to remeasure the three atmospheric parameters effective temperature, surface gravity, and $[\mathrm{Fe} / \mathrm{H}]$ metallicity.

Results. We find a very low $[\mathrm{Fe} / \mathrm{H}]$ dispersion along the isochrone $(0.07 \mathrm{dex})$, consistent with our error estimate $(0.05$ dex $)$. However, the $[\mathrm{Fe} / \mathrm{H}]$ trend is not reproducible. This shows that the data have the potential to reveal patterns of the magnitude of the expected physical effects, but the analysis methods need to be refined to cull systematic effects that currently dominate the patterns.
\end{abstract}

Key words. methods: data analysis - techniques: spectroscopic - stars: fundamental parameters

\section{Introduction}

The introduction of the wide-field integral field spectrograph MUSE (Bacon et al. 2010, 2014) mounted at UT4 (Yepun) of the ESO Very Large Telescope (VLT) recently ushered in a new area for the exploration of Galactic globular clusters. Husser et al. (2016, hereafter H16) carried out and analyzed observations of NGC 6397. With a field of one arcminute and a spatial resolution limited by the seeing, MUSE allows mapping a full globular cluster in a reasonable number of pointings. The instrument allows obtaining many more spectra in one shot than a multi-object spectrograph, and the continuous mapping of the area makes it possible to use deblending methods similar to those that are commonly applied for crowded-field photometry (e.g., DAOPHOT, Stetson 1987). Although these spectra do not have the high spectral resolution required for abundance analysis (Alves-Brito et al. 2012; Ernandes et al. 2018), they are suitable for determining the atmospheric parameters, effective temperature $\left(T_{\text {eff }}\right)$, surface gravity $(\log g)$, and metallicity $([\mathrm{Fe} / \mathrm{H}])$ using methods that are applicable at low resolution. Full-spectrum fitting is one of these methods, and its reliability and efficiency has been demonstrated in several instances. For example, Koleva \& Vazdekis (2012) have shown that the parameters can be retrieved from spectra with a resolution $R=\lambda / \Delta \lambda \approx 1000$, and the authors found that the precision was not degraded compared to high-resolution spectra or the appearance of significant biases. Full spectrum fitting offers the advantage of optimally

* Data are only available at the CDS via anonymous ftp to cdsarc.u-strasbg.fr (130.79.128.5) or via http://cdsarc. u-strasbg.fr/viz-bin/cat/J/A+A/635/A161 using the entire signal, which is particularly valuable in the present case, where the observations cover a range of apparent magnitudes and hence of signal-to-noise ratios $(\mathrm{S} / \mathrm{N})$.

H16 analyzed 18932 spectra of 12307 stars extracted from a mosaic of 23 MUSE pointings with full-spectrum fitting. The observations were compared to a grid of models (Husser et al. 2013, the Göttingen Spectral Library, GSL) that were computed with the PHOENIX synthesis code (Allard \& Hauschildt 1995) which was interpolated to minimize the residuals. One of the remarkable results of this work is the finding of a metallicity trend along the isochrone, with an amplitude of 0.2 to $0.3 \mathrm{dex}$. The metallicity is minimum at the turn-off (TO) and rises both along the sub-giant branch (sGB) and down the main sequence (MS). This is reminiscent of the expectations from atomic diffusion, which reduces the surface abundances of metals in regimes where mixing processes such as turbulence are relatively inefficient (Richard et al. 2002).

The mean observed effect in $\mathrm{H} 16$ is slightly stronger than found in the detailed abundance analysis of individual cluster stars of Nordlander et al. (2012), which was $\sim 0.1$ dex on average, but was based on a small number of stars in each of the evolutionary phases of interest. The authors of this study highlight the sensitivity of the metallicity trend to model aspects such as the efficiency of the turbulent mixing, temperature scale, nonlocal thermal equilibrium (NLTE) and 3D effects. The model grid used in H16 was computed with 1D models.

In addition, the magnitude of the detected metallicity variations are only a fraction of the mesh of the grid of available reference synthetic spectra. The GSL was computed at regular spacing in the $T_{\text {eff }}, \log g,[\mathrm{Fe} / \mathrm{H}]$, and $[\alpha / \mathrm{Fe}]$ parameter space, 
and the steps in metallicity are $1.0 \mathrm{dex}$ for $[\mathrm{Fe} / \mathrm{H}]<-2.0$ and $0.5 \mathrm{dex}$ for $[\mathrm{Fe} / \mathrm{H}]>-2.0$. Figure 1 (left panel) shows the distribution of the GSL spectra in the $\log g$ versus $T_{\text {eff }}$ and $[\mathrm{Fe} / \mathrm{H}]$ versus $T_{\text {eff }}$ diagrams, plotted over the distribution of the individual stars of the cluster. The mean metallicity of the cluster is close to the $[\mathrm{Fe} / \mathrm{H}]=-2.0$ dex slice of the GSL, and the whole metallicity range is well within the two surrounding slices, at -1.5 and -3.0 dex. We connot rule out that the measured trend is affected by the spline interpolation in the grid.

The potential significance of the result for our understanding of mixing processes in stars, may affect quantities of general interest in the study of star clusters, such as stellar lifetimes or the location of the TO. We therefore here revisit the result using a different approach. Studies of the integrated light of globular clusters have shown that the residuals of full spectrum fits are significantly lower when the artificial cluster spectra are based on empirical spectral libraries than when they are based on theoretical libraries (Martins et al. 2019).

We use two of these empirical spectral libraries to reanalyze the stellar spectra of the MUSE observations of NGC 6397. By using other reference spectra than $\mathrm{H} 16$, we introduce a different set of biases. Differences with the results of $\mathrm{H} 16$ will provide an assessment of systematic uncertainties, and the basis for future improvements.

Section 2 presents the data and their analysis, Sect. 3 our new analysis, Sect. 4 discusses the results, and Sect. 5 draws the conclusions.

\section{Data and earlier analysis}

The observations of a $5 \times 5$ mosaic $^{1}$ reaching out to a distance of $\sim 3.5$ arcmin from the cluster center were made during the MUSE commissioning, between 2014 July 26 to August 3 (ESO program identifier 60.A-9100(C)). They consisted of 127 pointings of exposure time of not more than $60 \mathrm{~s}$ to avoid saturation of the bright giant stars, for a total integration time of $95 \mathrm{~min}$. Each pointing covers a field of $1 \times 1$ arcmin with a spatial step of 0.2 arcmin, and the seeing was in the range $0.6^{\prime \prime}-1^{\prime \prime}$.

The data were reduced by the MUSE consortium using the official pipeline, and the extraction of the individual stellar spectra was described in H16, with a deblending approach presented in Kamann et al. (2013, implemented in the PampelMuse software, publicly available). This method relies on a photometric and astrometric input catalog, which in this particular case was the Anderson et al. (2008) table derived from images obtained with the Advanced Cmaera for Surveys (ACS) on board the Hubble Space Telescope (HST). An initial point spread function (PSF) model, set to be the seeing, is used to create a mock image that is cross-correlated with each wavelength layer of the MUSE cube to find the coordinates transformations. The most isolated and bright spectra are used to iteratively refine the PSF by subtracting all the other spectra. The final wavelengthdependent PSF is derived by smoothing the PSF obtained for each layer, and it is used to extract the spectra. In total, 18932 spectra of 12307 stars were extracted. The stars in the overlapping regions between the different fields were observed multiple times. The line spread function (LSF) varies with the wavelength from a full-width at half-maximum (FWHM) of about $2.82 \AA$ at $\lambda=4750 \AA$ (equivalent to an instrumental velocity dispersion, $\left.\sigma_{\text {ins }}=80 \mathrm{~km} \mathrm{~s}^{-1}\right)$ to about $2.54 \AA$ at $\lambda=7000 \AA$ $\left(\sigma_{\text {ins }}=46 \mathrm{~km} \mathrm{~s}^{-1}\right)$, and stays approximately constant further in

1 Two of the planned fields, located at the periphery, were not observed; 23 fields are available. the red. The actual LSF also varies across the 24 spectrographs forming MUSE by roughly $0.1 \AA$.

Out of the 18932 extracted spectra, 14271, with an S/N greater than 5 are distributed on the MUSE website ${ }^{2}$ (the raw data are available in the ESO archive $^{3}$ ).

To determine the atmospheric parameters, H16 proceeded in three steps. First they obtained $T_{\text {eff }}$ and $\log g$ by fitting the HST photometry to an isochrone. They then used these parameters to generate interpolated models that they cross-correlated with the observations to derive the radial velocity $\left(v_{\text {rad }}\right)$. In the third step, they performed an optimization to produce the final $T_{\text {eff }},[\mathrm{Fe} / \mathrm{H}], v_{\text {rad }}$, line broadening, and telluric absorption spectrum. They used their own full-spectrum fitting procedure, using GSL interpolated by cubic spline as reference. The interpolation cannot be a simple linear interpolation because the optimization method requires its derivative to be continuous. The value of $\log g$ was not optimized; the adopted value is the photometric one. The authors justify this choice as a precautionary approach to prevent a possible degeneracy between $\log g$ and the broadening.

This analysis provided parameters for 5882 spectra of 4132 stars with an $S / N>20$, above which the formal error on $T_{\text {eff }}$ is lower than $100 \mathrm{~K}$, and on $[\mathrm{Fe} / \mathrm{H}]$ lower than 0.16 dex. The tables with results were kindly provided to us by Tim-Oliver Husser. For 367 of these spectra $\log g$ was not determined, and consequently, neither $T_{\text {eff }}$ nor $[\mathrm{Fe} / \mathrm{H}]$ were measured. To clean the sample of the nonmember stars, we selected the spectra within an ellipse centered on $v_{\text {rad }}=17.8 \mathrm{~km} \mathrm{~s}^{-1}$ and $[\mathrm{Fe} / \mathrm{H}]=-1.96 \mathrm{dex}$ (mean parameters of the cluster), and semiaxes of $29 \mathrm{~km} \mathrm{~s}^{-1}$ and 1.08 dex (corresponding approximately to the selection area measured in Fig. 7 of H16; the actual values are not reported in the text of this paper). The sample is reduced to 5510 spectra of 4089 distinct cluster members. Furthermore, we are interested here in the TO and red giant branch (RGB), and because the method of determining the stellar parameters by full-spectrum fitting has never been validated for the hot stars, we followed $\mathrm{H} 16$ and limited the sample to $T_{\text {eff }}<7000 \mathrm{~K}$. This criterion excludes the hot horizontal branch stars. Finally, the sample contains 5400 spectra of 4053 distinct cluster members.

\section{Reanalysis}

\subsection{Method}

The aim of this paper is to repeat H16's analysis of the metallicity trend along the cluster sequence with other reference libraries in order to determine the reliability of this trend. Alternatives to GSL are the ELODIE and MILES empirical libraries, among others. Synthetic and empirical libraries are seen as complementary; each has their advantages and drawbacks. On the one hand, synthetic spectra can be computed at any point in the parameter space and are therefore not limited to the region populated with stars that can be observed (i.e., essentially stars from the solar neighbourhood). They are also free of noise and observational signatures, and their spectral resolution can be as high as necessary. However, synthetic spectra still fail to accurately match observed spectra, because the physical models and lists of atomic and molecular transitions are limited (Martins et al. 2014). On the other hand, empirical libraries can accurately reproduce observed spectra, and this is why they are extensively used to model integrated spectra of stellar populations (Martins et al. 2019).

\footnotetext{
http://muse-vlt.eu/science/globular-cluster-ngc-6397 http://archive.eso.org/
} 


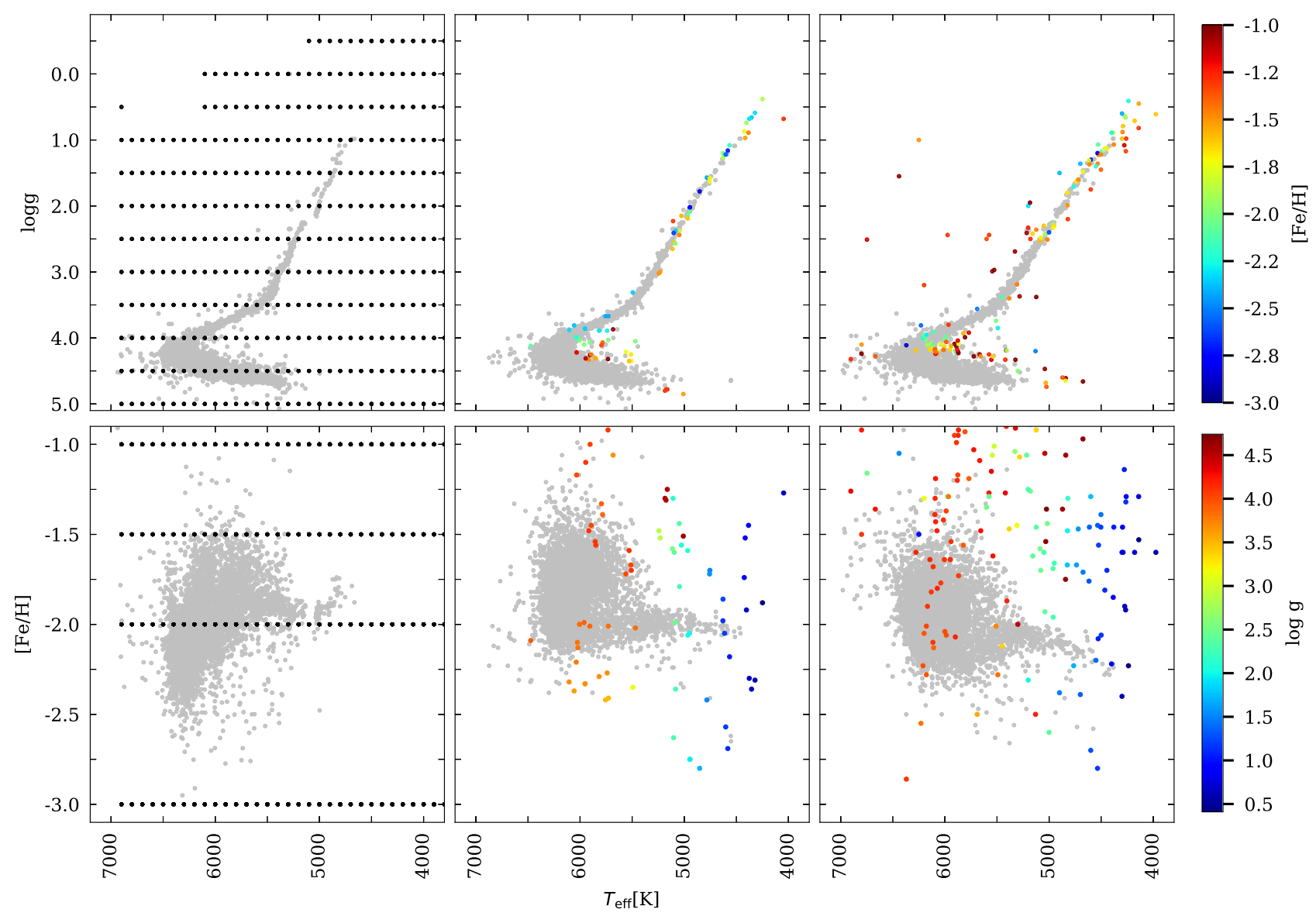

Fig. 1. Distribution of the library spectra in the region of the parameter space occupied by the cluster stars. The gray dots represent the H16 measurements of the cluster members. The top graphics are the projections in the $\log g$ vs. $T_{\text {eff }}$ plane and the bottom graphics show the [Fe/H] vs. $T_{\text {eff }}$ projections. The overplotted colored points are the location of the reference spectra in the three libraries used in this paper. Left panel: Göttingen Spectral Library used by H16, central panel: ELODIE library, and right panel: MILES library. These last two libraries were used in tour reanalysis. Only the library stars corresponding to the region mapped by the our sample are shown: $T_{\text {eff }}<7000 \mathrm{~K}$, and $-3.04<[\mathrm{Fe} / \mathrm{H}]<-0.88 \mathrm{dex}$ (77 stars for ELODIE and 137 for MILES, including 40 and 46 stars, respectively with $[\mathrm{Fe} / \mathrm{H}]<-1.7 \mathrm{dex}$ ). The color scales for the ELODIE and MILES libraries are indicated on the right.

ELODIE is an empirical library, initially presented in Prugniel \& Soubiran (2001) and later upgraded to increase the coverage in the parameter space. We used the latest version (version 3.2), described in Wu et al. (2011), containing 1962 spectra at a constant FWHM resolution of $0.55 \AA$ over the wavelength range $3900-6800 \AA(R \approx 10000)$. The second library, MILES (Sánchez-Blázquez et al. 2006), contains spectra of 985 stars at a resolution about $2.55 \AA(R \approx 2000)$ in the wavelength range $3536-7410 \AA$. The distribution of the stars populating the region of the cluster in the parameter space are represented in the central and right panels of Fig. 1 for ELODIE and MILES, respectively. Both libraries sample the region of the RGB reasonably well down to almost the TO. Along the MS, most of the stars are on the high-metallicity side compared to the cluster, and only a few have the cluster metallicity. It is also noticeable that the library MSs are shifted toward lower gravities by about 0.1 dex compared to the cluster star gravities determined by isochrone fitting. We found indications that this may be at least partly due to an underestimate of the gravities in the libraries.

The fitting procedure to be used for estimating stellar parameters requires interpolating through the libraries to compute a spectrum for any set of $T_{\text {eff }}, \log g$, and $[\mathrm{Fe} / \mathrm{H}]$. For this purpose, we used polynomial interpolators computed using stellar parameters of the library stars compiled from the literature, generally obtained through detailed abundance studies using high-resolution spectroscopy. The ELODIE interpolator is described in Wu et al. (2011), and for MILES, we used the interpolator described in Sharma et al. (2016). The quality of the interpolated spectra naturally depends on the distribution of the stars in the parameter space: the denser the distribution, the better the interpolation. The quality also depends on the accuracy of the input stellar parameters that were used when the interpolators were computed, and on the intrinsic accuracy of the interpolators. At the margin of the parameter space, the interpolators use synthetic spectra to extend the validity range, but with a lower quality. The MS of the cluster is at the edge of the validity region of the interpolators. However, using the procedure described below, we successfully fit synthetic spectra from Coelho et al. (2005) and from the GSL that were chosen to map this regime. This gave us some confidence in our ability to analyze MS spectra. However, similar tests with the cluster's spectra failed to restore the correct gravity. The solutions were found at a correct temperature, but located on the giant branch. The reasons for this difficulty are not completely clear, but are probably related to the drop in $\mathrm{S} / \mathrm{N}$ from $\sim 60$ near the $\mathrm{TO}$ to $\sim 20$ on the lower MS, and to stronger contamination by other cluster stars for the fainter objects. We here prefer to restrict the spectral 
analysis to $\log g<4.2$ and high $\mathrm{S} / \mathrm{N}$. and are subject to a strong light contamination by the other cluster stars. Although we here follow H16 and adopt the isochrone gravities, we prefer to restrict our analysis to the RGB, $\log g<4.2$. This sample contains 1587 spectra of 1063 stars.

The atmospheric parameters were determined with the ULySS package (Koleva et al. 2009) by minimizing the squared residuals between the MUSE observation and an interpolated spectrum,

$S(\lambda)=P_{n}(\lambda) \times G\left(v_{\text {rad }}, \sigma_{\text {rel }}\right) \otimes \operatorname{TGM}\left(T_{\text {eff }}, \log \mathrm{g},[\mathrm{Fe} / \mathrm{H}], \lambda\right)$,

where $P_{n}(\lambda)$ is a series of Legendre polynomial up to degree $n$, meant to absorb the instrumental spectral response and line-ofsight extinction; we used $n=20 . G\left(v_{\text {rad }}, \sigma_{\text {rel }}\right)$ is a Gaussian function centered at $v_{\text {rad }}$ and with standard deviation $\sigma_{\text {rel }}$. The spectra were binned in logarithmic wavelength, so that the Doppler shift can be expressed by a convolution. TGM is the spectral interpolator. The free parameters are $T_{\text {eff }},[\mathrm{Fe} / \mathrm{H}], v_{\text {rad }}, \sigma_{\text {rel }}$ and the coefficients of $P_{n}(\lambda)$. See Arentsen et al. (2019) for other details on the procedure.

$\sigma_{\text {rel }}$ was let free in order to account for the variation of instrumental broadening from spectrum to spectrum (as described in Sect. 2, the MUSE LSF varies between the individual spectrographs). The relative LSF between MUSE and the libraries changes significantly over the wavelength range, and we therefore ingested this variation pattern in the interpolated spectra, before the minimization. This was made with a stepwise convolution of the library (see the ULY_LSF_CONVOL function in the ULySS package). The LSF injection improves the quality of the fits and slightly reduces the errors of the estimated parameters, but it does not affect the general trends.

In the case of the ELODIE library, the injected LSF was chosen so that the interpolated spectrum has a higher resolution than the MUSE spectrum. The observation was then fit by letting $\sigma_{\text {rel }}$ free. For the MILES library, the resolution after the LSF injection is very close to that of MUSE, but because of the spectrumby-spectrum variations by $0.1 \AA$, it is sometimes lower. In such cases, detected because the first fit fails to determine $\sigma_{\text {rel }}$, we convolved the observation with a Gaussian with a dispersion of $30 \mathrm{~km} \mathrm{~s}^{-1}$, and then let the fitting procedure determine $\sigma_{\text {rel }}$.

A difference between the present procedure and that of H16 is that the latter adjusted the telluric absorption together with the stellar parameters and broadening. Telluric absorption features are prominent in the red part of the spectra, which are outside the currently analyzed region, and we more simply masked (i. e., ignored) the two strongest features. The first feature is the water absorption near the $\mathrm{NaD}$ line that affects both the ELODIE and MILES, and the second is the B band, near $6887 \AA$ (Fraunhofer 1817, 1821), which affects MILES.

No error spectra are associated with the extracted observations, but a mean $\mathrm{S} / \mathrm{N}$ is provided. We used it to determine a noise level that we assumed to be identical throughout the spectrum, meaning that all wavelength bins have the same weight. This assumed error sets the scale of the fitting errors on the parameters that we tune in Sect. 3.2 into internal errors, by matching the repeated observations of stars in the overlaps of the different pointings.

In order to keep the reanalysis as closely as possible to that of $\mathrm{H} 16$, we carried it out by fixing $\log g$ to their photometric value (but see Sect. 4.2, where we also determine $\log g$ spectroscopically). As first guesses, we used for $T_{\text {eff }}$, the photometric value, and for $[\mathrm{Fe} / \mathrm{H}]$ we used $-2.0 \mathrm{dex}$, which is an approximation of the cluster metallicity.

\subsection{Assessment of the internal errors}

The full catalog contains 5510 measurements for 4089 stars (including the stars from the MS to the RGB). For the 1200 stars with two or more repeated observations, the spectra differ by the noise and by the propagation of systematics introduced by the spectrograph and data reduction. We assessed the precision by comparing the atmospheric parameters measured on each pair of observations as in Arentsen et al. (2019). We computed for each pair of observations $i$ :

$\Delta P_{i}=\frac{P_{1, i}-P_{2, i}}{\sqrt{\epsilon_{1, i}^{2}+\epsilon_{2, i}^{2}}}$,

where $P$ can be $T_{\text {eff }}$ or $[\mathrm{Fe} / \mathrm{H}], \epsilon$ is the formal ULySS error on the respective parameters, and 1 and 2 indicate the observation of each spectrum of the pair. This distribution is expected to be nearly Gaussian, and if the errors are properly scaled, their standard deviation is expected to be one. For both parameters we find $\Delta \mathrm{P}=2.2$ and 1.8 for ELODIE and MILES, respectively. Because we assumed a constant noise scaled to produce the average $\mathrm{S} / \mathrm{N}$ determined by the data reduction and spectrum extraction pipeline, we indeed expected deviations from unity. We also assumed that all the pixels are independent, which cannot be the case because the spectra were rebinned into evenly sampled wavelength (this process necessarily introduces a correlation in the noise), and this leads to an underestimation of the errors.

We verified that these distributions were similar throughout the entire parameter space, and we found only a marginal indication that they may depend on $\mathrm{S} / \mathrm{N}$, in a way consistent with an additional source of noise independent of $\mathrm{S} / \mathrm{N}$. The effect is about $15 \mathrm{~K}$ and 0.01 dex to be quadratically added on the errors on $T_{\text {eff }}$ and $[\mathrm{Fe} / \mathrm{H}]$,respectively. This is very small.

The fact that the distributions are similar for the two parameters is a satisfactory sanity check of our analysis, and we did not investigate the deviations from unity further because they are not relevant for our present discussion. We rescaled the errors by the factors described above when we computed the errors in the final table (see Sect. 3.4).

\subsection{Assessment of the external errors (accuracy)}

For an estimate of the external errors, we compared our measurements with previous works. First of all, the comparison of 1587 measurements with $\mathrm{H} 16$ indicates $T_{\text {eff }}(\mathrm{H} 16)-T_{\text {eff }}$ (ELODIE/MILES $)=$ $92 \mathrm{~K}$ with a dispersion of $40 \mathrm{~K}$. For the metallicity, the bias is different for ELODIE or MILES: $[\mathrm{Fe} / \mathrm{H}](\mathrm{H} 16)-[\mathrm{Fe} / \mathrm{H}](\mathrm{ELODIE})=$ $-0.07 \mathrm{dex}$, and $[\mathrm{Fe} / \mathrm{H}](\mathrm{H} 16)-[\mathrm{Fe} / \mathrm{H}]($ MILES $)=0.03$, with a dispersion of 0.15 dex. Because the three series used the same reduced spectra, they can be similarly affected by systematics due to the data. These figures were therefore be regarded as lower limits to the errors, and comparisons with independent measurements will give a more complete assessment of the external errors.

Five previous studies determined the parameters of a significant number of stars in this cluster. Carretta et al. (2009a) measured 13 stars at high spectral resolution, with the UVES spectrograph attached to the VLT, and Carretta et al. (2009b) measured 144 stars at intermediate resolution, with GIRAFFE on the same telescope, all giants with $T_{\text {eff }}<5400 \mathrm{~K}$. Lovisi et al. (2012) measured 146 stars, but those in common with the MUSE sample are mostly blue stragglers, which are not discussed here. Finally, Korn et al. (2007), and Lind et al. (2008) observed stars 


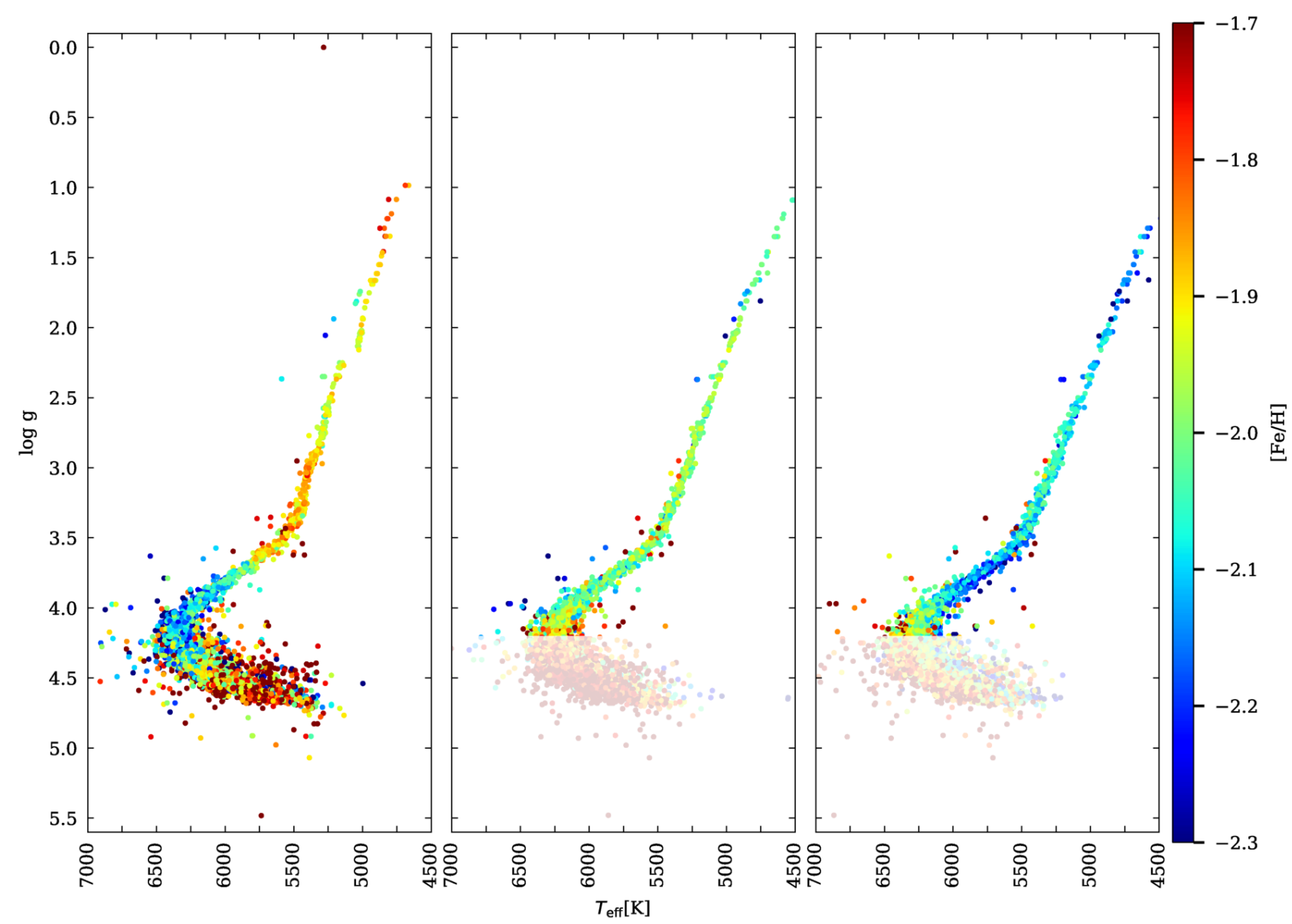

Fig. 2. Distribution of the parameter measurements for the 5400 spectra of cluster members with good-quality spectra in the log $g$ vs. $T_{\text {eff }}$ plane. Left panel: H16 catalogue, central and right: our reanalysis with the ELODIE and MILES interpolators, respectively. The color scale, shows the metallicity, in the right margin. The region of the diagram that we do not discuss here $(\log g<4.2)$ is shown with paler colors.

with the UVES (18 stars) and GIRAFFE (116 stars) spectrographs, respectively, but in more peripheral fields that lie outside the MUSE pointings.

The comparisons with the two first datasets are very similar, so that we can merge them and report only the total comparison. We find $T_{\text {eff }}($ lit $)-T_{\text {eff }}$ (ELODIE) $=15 \mathrm{~K}$ with a dispersion of $30 \mathrm{~K}$, and $T_{\text {eff }}($ lit $)-T_{\text {eff }}$ (MILES) $=48 \mathrm{~K}$ with a dispersion of $25 \mathrm{~K}$. For the metallicity, we find no differences with ELODIE, and $[\mathrm{Fe} / \mathrm{H}](\mathrm{lit})-[\mathrm{Fe} / \mathrm{H}]($ MILES $)=0.07 \mathrm{dex}$, in both cases with a dispersion of 0.05 dex.

The comparison between the H16 measurements and the literature displays the following differences: $T_{\text {eff }}($ lit $)-T_{\text {eff }}(\mathrm{H} 16)=$ $-100 \mathrm{~K}$ and $[\mathrm{Fe} / \mathrm{H}]($ lit $)-[\mathrm{Fe} / \mathrm{H}](\mathrm{H} 16)=-0.08 \mathrm{dex}$, with dispersions of $48 \mathrm{~K}$ and $0.06 \mathrm{dex}$ on the two parameters. In all cases the $\log g$ values are very consistent with the literature, which is expected because they were determined photometrically in all cases with very similar methods.

The picture that emerges from these comparisons is that (i) $T_{\text {eff }}$ measured by $\mathrm{H} 16$ is on average hotter than the literature and our own measurements. (ii) $[\mathrm{Fe} / \mathrm{H}]$ is reasonably consistent between the different series; our measurements are marginally more consistent with the literature than those of H16. (iii) The consistency is similar for our two analyses, with a marginally better performance of ELODIE.

For the observations compared with the literature, the mean estimated internal errors after the rescaling described in Sect. 3.2 are 18 and $25 \mathrm{~K}$ on $T_{\text {eff }}$, and 0.04 and $0.05 \mathrm{dex}$ on $[\mathrm{Fe} / \mathrm{H}]$ for
ELODIE and MILES, respectively, which is about 1.25 times lower than the external dispersions estimated here. Because these dispersions include both the errors on our and on the literature measurements, we conclude that our estimates of the internal errors properly reflect the external errors, or accuracy of the measurements.

\subsection{Results}

Our measurements of the atmospheric parameters for the 1587 spectra are available in electronic form at the CDS.

Figure 2 presents the distribution of the measurements in the $\log g$ versus $T_{\text {eff }}$ plane. The vertical axis is the photometric gravity, and the horizontal axis is the $T_{\text {eff }}$ from H16, ELODIE, and MILES, for the left, central, and right panel, respectively. The data points are color-coded according to the metallicity. This can be compared with the Fig. 8 of H16. The RGB harbors an unphysical break in the $\mathrm{H} 16$ data near $T_{\text {eff }}=5000 \mathrm{~K}$. We cannot provide a final explanation for this break, but it may correspond to a discontinuity of the opacities used for GSL, at a threshold temperature where some molecular bands are considered. This RGB is generally hotter than in our analysis, reflecting the systematic difference quantified in Sect. 3.3 that was also found when $\mathrm{H} 16$ was compared to earlier measurements. The small $T_{\text {eff }}$ difference between ELODIE and MILES is consistent with the uncertainties on the temperature calibration of the RGB. 
Figure 3 shows the distribution of the sample in the $[\mathrm{Fe} / \mathrm{H}]$ versus $T_{\text {eff }}$ plane. The top panel shows the result from the H16 analysis, and the middle and lower panels are the results with the ELODIE, and MILES interpolators respectively. In the three cases, the low dispersion around the observed trend reveals a high internal consistency. The mean $[\mathrm{Fe} / \mathrm{H}]$ dispersion around the cluster isochrone, estimated as the average rms computed in the $T_{\text {eff }}$ bins shown in Fig. 3, is about 0.07 dex. Compared to the 0.05 dex external error reported in Sect. 3.3, we see no clear indication of a cosmic dispersion.

In addition to this internal consistency, the three analyses display different patterns of metallicity variation. The results with ELODIE would be consistent with a constant metallicity. The H16 metallicity steadily increases along the sGB, while the MILES trend is wavy, with a pronounced $[\mathrm{Fe} / \mathrm{H}]$ decline immediately above the TO.

\section{Discussion}

\subsection{Changes in surface metallicity above the turn-off}

Considering that chemical tagging (the ability to associate individual stars with their birth environment) has been a science driver for many of the large surveys now underway, the ability to differentiate surface from initial abundances is a crucial necessity of stellar evolution models. Many processes can alter the surface abundance of a star during its lifetime. Atomic diffusion is one of the most frequent discussed processes. It encompasses different processes that operate in the radiative regions of the stars and cause the redistribution of elements, mainly by the migration of heavier elements toward its center, which consequently lowers the surface metallicity of the stars. Although atomic diffusion in stars has been theoretically predicted more than a century ago by Chapman (1917), its real effect on stellar evolution remains debated (VandenBerg et al. 2002). Mixing processes (e.g., convection and turbulent mixing), compete with gravitational settling to weaken the actual depletion in heavy elements. The efficiency of these processes, at variance with the pure atomic diffusion, has no well-established physical description, and can only be determined through comparison with observations. For instance, Dotter et al. (2017) investigated models with and without these effects and found that the differences in ages obtained through isochrones might reach up to $20 \%$. Star clusters, with a coeval stellar population that in principle is initially chemically homogeneous, are the perfect laboratory for testing the balance between the different phenomena.

It is expected that because of atomic diffusion, the surface abundance of heavy element is minimum at the TO, increasing both down the MS and toward the RGB. Metallicity trends near the TO of globular clusters have previously been discussed in NGC 6752 by Gruyters et al. (2013), and in M30 Gruyters et al. (2016), and in the M67 open cluster by Bertelli Motta et al. (2018), Souto et al. (2018), Gao et al. (2018), and Liu et al. (2019). In particular Gratton et al. (2001) and more recently Lovisi et al. (2012) found no difference between the abundances of TO stars and base-RGB stars for NGC 6397.In contrast, Korn et al. (2007), Lind et al. (2008) and Nordlander et al. (2012) found a clear trend. The latter three works found similar trends for the iron metallicity from the TO to the RGB, obtaining maximum metallicity differences of about $0.15-0.20$ dex. They argued that their results indicate the need of atomic diffusion with weak efficiency of turbulent mixing, but also that, based on the uncertainties from modeling techniques, it cannot be ruled out that this might be just an

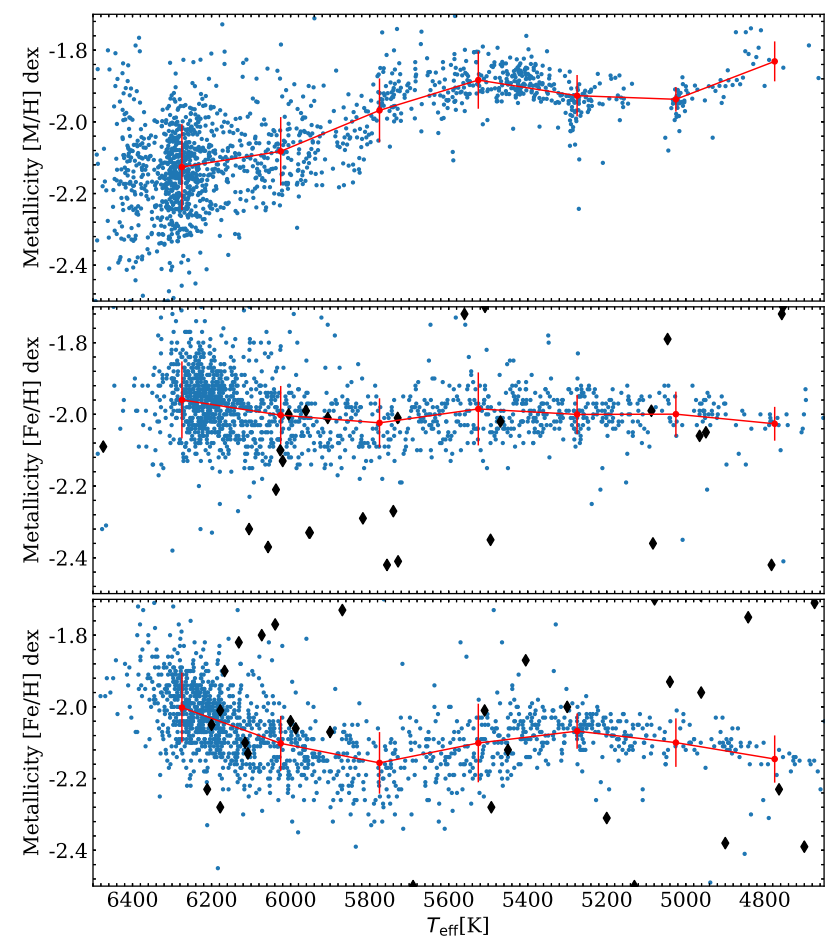

Fig. 3. $[\mathrm{Fe} / \mathrm{H}]$ vs. $T_{\text {eff }}$ for the giants $(\log g<4.2$ dex $)$. Top panel: $\mathrm{H} 16$ catalog, middle and bottom panels: our reanalysis with the ELODIE and MILES interpolators, respectively (blue dots). The solid red line connects the mean values and standard deviations of the metallicity in $250 \mathrm{~K}$ wide bins. The black dots show the location of the ELODIE and MILES library stars, in the middle and bottom panel, respectively. The mean $[\mathrm{Fe} / \mathrm{H}]$ dispersion in $[\mathrm{Fe} / \mathrm{H}]$ of stars taken in bins of $250 \mathrm{~K}$ for $\mathrm{H} 16$ is 0.076 , for ELODIE is 0.075 and that for MILES is 0.079 calculated for stars with $T_{\text {eff }}<6500 \mathrm{~K}$.

artifact. For example, the Fig. 7 of Lind et al. (2008) shows that although they and Korn et al. (2007) found a very similar trend in $[\mathrm{Fe} / \mathrm{H}]$ along the Hertzsprung-Ruseel diagram, the systematic differences between their values is about 0.15 dex, which they explained as possible differences between the NLTE corrections. In other clusters, the measured amplitude of variations is smaller, or no effect was detected. All these works attempted to determine chemical abundances with dedicated techniques, addressing and trying to take into account model weaknesses such as the NLTE and $3 \mathrm{D}$ effects.

We here found different $[\mathrm{Fe} / \mathrm{H}]$ trends above the TO using three different stellar libraries. This casts some doubt on the reality the reality of the detection of atomic diffusion effects.

Regarding the H16 results, we recall that the magnitude of the trend is significantly smaller than the GSL grid mesh and that the synthetic spectra themselves can introduce systematics due to calculation details. The nonuniform distribution of the cluster stars along the sequence in the top panel of Fig. 3 is an indication that some finite-grid effects are indeed present along the temperature axis in this analysis, but the ramification for other parameters remain unclear. In contrast, the ELODIE and MILES interpolators do not suffer from this wide-mesh drawback, but they are limited by the small number of stars in this region of the parameter space (about 40 stars for both libraries in the \pm 0.3 dex slice around the cluster metallicity, see Fig. 3). These observed library spectra are also subject to noise and to the effect of peculiarities of individual stars. In well-populated regions of the parameter space (typically close to solar metallicity), these effects are averaged out because a fair number of stars 


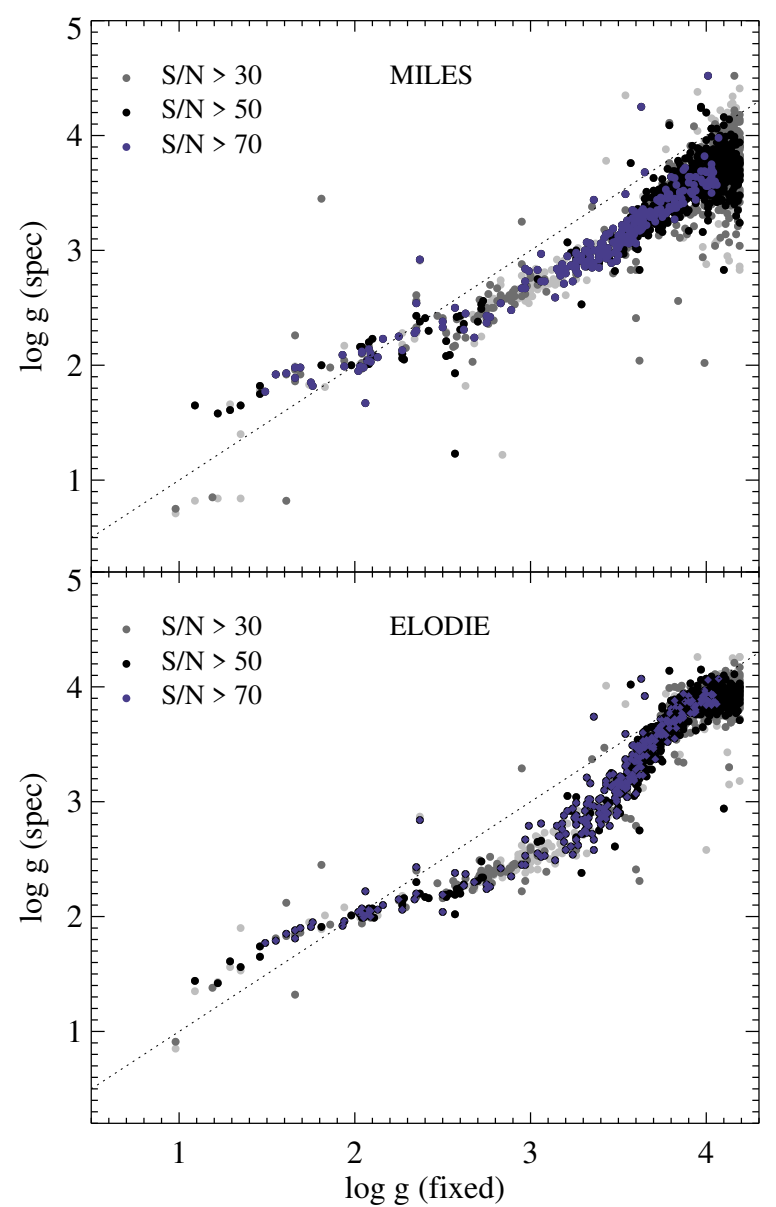

Fig. 4. Comparison between the spectroscopic and photometric $\log g$. The dotted line is the 1:1 correspondence, the color of the symbol reflects $\mathrm{S} / \mathrm{R}$ as indicated in the figure. Top panel: MILES analysis and bottom panel: that of ELODIE.

have similar parameters, but in this low-metallicity regime, every single star might have a strong influence on the interpolator.

The distribution of the ELODIE stars (the black dots in Fig. 3) is uniform except in the region $T_{\text {eff }}>6100 \mathrm{~K}$, which includes only one star with $[\mathrm{Fe} / \mathrm{H}]<-1.7$ dex. In the case of MILES, we note that in the range $5500<T_{\text {eff }}<5850 \mathrm{~K}$ one library star has $[\mathrm{Fe} / \mathrm{H}]<-1.7 \mathrm{dex}, \mathrm{HD} 140283$, with $[\mathrm{Fe} / \mathrm{H}] \approx$ -2.57 dex. The MILES pattern in this regime may therefore be an artifact of the interpolation. The interpolator we used here is an improvement on the interpolator that was initially published by Prugniel et al. (2011). The improvement concerned the cool stars $\left(T_{\text {eff }}<4800 \mathrm{~K}\right)$, and consisted of refining the stellar parameters by critically scrutinizing the literature and tuning the interpolator. The number of terms in the polynomial development was also increased, essentially to reflect the variation between the giants and the dwarfs better, and this can affect its behavior at warmer temperature. We repeated the analysis with this first version interpolator, in order to verify the reliability of the metallicity trend with respect to the change of the interpolator, and we did not notice any significant difference. During the preparation of this improved MILES interpolator, a series of interpolators were constructed, where for each a single star was excluded. They are called X-interpolators in Sharma et al. (2016), and they were used to verify the stability of the results. We repeated the analysis here with all the $\mathrm{X}$-interpolators of the library stars in the region of the parameters we analyzed to determine whether the trend was modified for some of these stars. We found the

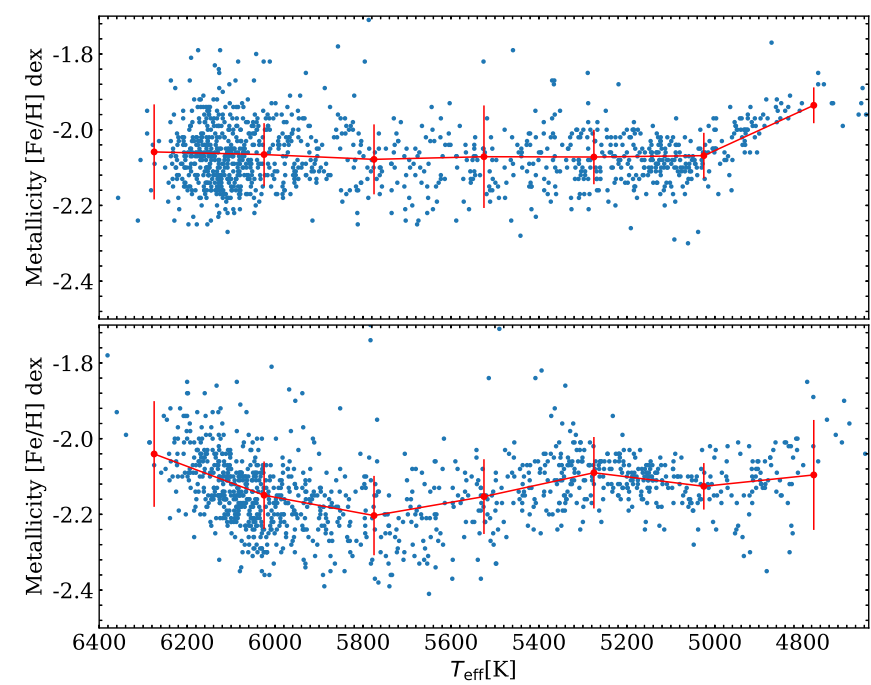

Fig. 5. $[\mathrm{Fe} / \mathrm{H}]$ vs. $T_{\text {eff }}$ for the giants $(\log g<4.2$ dex), with $\log g$ determined spectroscopically. Top panel: results obtained with the ELODIE interpolator, and bottom panel: those obtained with the MILES interpolator. The solid red line connects the mean values and standard deviations of the metallicity in $250 \mathrm{~K}$ wide bins. The difference with Fig. 3 is that $\log g$ was this time determined by the full-spectrum fitting, not by photometry.

trend to be robust, and therefore rule out any strong bias due to an individual star. We also compared the parameters that were used to compute the MILES interpolator with an updated compilation of the literature, and did not find any discrepancy. Finally, we redetermined the parameters of the MILES spectra by fitting in the same way as the MUSE observation (self-inversion of the interpolator), and we found no systematic effect there either.

The difference between the trends found for the three analyses remains to be understood. Each analysis approach has its merits and drawbacks, and we found no reason prefer one more that the others.

\subsection{Spectroscopic surface gravity}

H16 did not fit $\log g$ from the spectra because of concerns of degeneracy between the instrumental broadening and the surface gravity. Because the MUSE LSF cannot be precisely assessed for the spectra extracted from the final cubes, the authors preferred to adopt $\log g$ from their photometric fit to the isochrone. Although the line width is the main indicator for the gravity in individual spectral lines that are observed at high spectral resolution, the degeneracy between the broadening and the gravity may not be important at medium resolution and for full-spectrum fitting, where all the lines are used, notwithstanding their sensitivity to gravity.

To determine the effect of the gravity on the results for the metallicity, we performed a separate analysis with $\log g$ as an additional free parameter. Figure 4 compares the two values. For the high $\mathrm{S} / \mathrm{N}$ spectra, $(S / N>50)$, the differences in $\log g$ do not exceed 0.5 dex between the two methods. When we compare the new values with literature values (see Sect. 3.3), we find log $g($ lit $)-\log g($ spectro $)=0.15$ dex, with a dispersion of 0.25 dex. $T_{\text {eff }}$ obtained with free $\log g$ is lower by $\sim 40 \mathrm{~K}$ (ELODIE) and $85 \mathrm{~K}$ (MILES) compared to the values when $\log g$ was fixed.

The $[\mathrm{Fe} / \mathrm{H}]$ trends with this analysis, presented in Fig. 5, are not significantly modified in this new analysis (compare with Fig. 3). 


\section{Conclusions}

We repeated the analysis of 1587 MUSE spectra of 1063 stars located above the TO of NGC 6397 that was originally performed by H16. While their analysis used a grid of synthetic stellar spectra, we have used two empirical stellar libraries, ELODIE and MILES, to determine $T_{\text {eff }}$ and $[\mathrm{Fe} / \mathrm{H}]$ of these stars. These spectra have $S / N \gtrsim 50$, and comparisons with $\mathrm{H} 16$ and earlier studies suggest that the errors in our determinations are $\sim 25 \mathrm{~K}$ and 0.05 dex on the two parameters respectively. The metallicity dispersions of the measurements, $\sim 0.07$ dex, agree with these figures.

Despite this fair consistency, the metallicity trends along the sGB and RGB are different in the three analyses. No trend was found with ELODIE, and the trend found with MILES does not match the theoretical expectations. Previous observational studies of this and other clusters also lead to contradictory results about the reality of this variation, and the detection of the effect of atomic diffusion.

These empirical libraries contain only a limited number of stars in this low-metallicity regime (they do not have enough stars on the MS, which makes it impossible to explore the trend below the TO). Despite this limitation, the empirical libraries are essential for validating the synthetic libraries, and they are still required ingredients for models of integrated spectra of stellar populations as are used in extragalactic astronomy. Therefore, the motivation to continue improving the empirical libraries remains strong. We here clearly identified the regime of $[\mathrm{Fe} / \mathrm{H}] \sim-2$ as an area where the libraries deserve to be improved.

The metallicity trend found by $\mathrm{H} 16$ qualitatively reproduces the expected effect of atomic diffusion. The reference grid of synthetic spectra has a wide metallicity mesh, however, which casts doubts on the capability of resolving the small physical effect. When the grid mesh are compared to the generally accepted accuracy in the determinated atmospheric parameters, most of the grids that are currently used for population analysis are too coarse in their metallicity coverage. For $T_{\text {eff }} \sim 6000 \mathrm{~K}$, the mesh size typically is $200 \mathrm{~K}$, which is roughly four times the admitted precision on measurements. On $\log g$ the size is $0.5 \mathrm{dex}$, which about five times the precision, and on $[\mathrm{Fe} / \mathrm{H}]$, the size is $0.5 \mathrm{dex}$, which is about ten times the precision. For example, for the GSL the meshes sizes are $100 \mathrm{~K}, 0.5$, and $0.5 \mathrm{dex}$, and in Coelho (2014) they were $200 \mathrm{~K}, 0.5$, and 0.5 dex on the three parameters. Only recently have libraries with a finer metallicity sampling become available. For example, INTRIGOS (Franchini et al. 2018) and Allende Prieto et al. (2018) have mesh sizes of $250 \mathrm{~K}, 0.5$, and 0.25 dex. Adopting finer sampling is naturally challenging in terms of both the computing time required to produce the large number of spectra, and the data volume which needs to be manipulated, which is still in the 100s GB in Allende Prieto et al. (2018). To properly resolve the metallicity trends due to atomic diffusion, or to determine the other surface abundances alterations due to other processes, a mesh size of 0.1 dex is required.

From point of view of high resolution abundance analysis, there is also large space for improvement. Limitations such as the treatment of NLTE and 3D effects are still a large source of uncertainty.

We illustrated the limits of the current libraries at low metallicity. While observations of Galactic globular clusters are classical benchmark for population models, the empirical libraries are at best limited near the TO of these clusters, and the interpolated spectra used for the models are crudely approximate. The most recent library is the X-shooter Spectral Library, XSL
Arentsen et al. (2019). It has a more uniform coverage of the metallicity range than earlier libraries, but also suffers from a relative shortage of TO and MS stars in the low metallicity regime. For the stellar population models and for the validation of synthetic spectra, it is desirable to extend the coverage of these libraries.

Acknowledgements. We thank Tim-Oliver Husser who shared with us the tables with the results of the H16 analysis, and the MUSE team for making public the extracted spectra on their web site. We acknowlege the LABEX Lyon Institute of Origins (ANR-10-LABX-0066) of the Université de Lyon for its financial support within the program "Investissements d'Avenir" (ANR-11-IDEX0007) of the French government operated by the National Research Agency (ANR), and IDEXLYON for their support fo the Indo-French Astronomy school (IFAS4), where this project was started. This work was supported by the Programme National Cosmology et Galaxies (PNCG) of CNRS/INSU with INP and IN2P3, co-funded by CEA and CNES. We thank Yasna Ordenes, Yue Wu, Jean Damascéne Mbarubucyeye, Sina Chen, Sonali Borah, Raghu Prasad, and Saumya Gupta, who contributed to early investigations on this project. We thank the referee for the comments, which helped improve the quality of the paper. L. P. M. thanks FAPESP (grant 2018/26381-4) and CNPQ (grant 306359/20189). L. P. M. also thanks L. Tresse, director of CRAL, for the hosting during the development of this work.

\section{References}

Allard, F., \& Hauschildt, P. H. 1995, ApJ, 445, 433

Allende Prieto, C., Koesterke, L., Hubeny, I., et al. 2018, A\&A, 618, A25

Alves-Brito, A., Yong, D., Meléndez, J., Vásquez, S., \& Karakas, A. I. 2012, A\&A, 540, A3

Anderson, J., Sarajedini, A., Bedin, L. R., et al. 2008, AJ, 135, 2055

Arentsen, A., Prugniel, P., Gonneau, A., et al. 2019, A\&A, 627, A138

Bacon, R., Accardo, M., Adjali, L., et al. 2010, in Ground-based and Airborne Instrumentation for Astronomy III, Proc. SPIE, 7735, 773508

Bacon, R., Vernet, J., Borisova, E., et al. 2014, The Messenger, 157, 13

Bertelli Motta, C., Pasquali, A., Richer, J., et al. 2018, MNRAS, 478, 425

Carretta, E., Bragaglia, A., Gratton, R., \& Lucatello, S. 2009a, A\&A, 505, 139

Carretta, E., Bragaglia, A., Gratton, R. G., et al. 2009b, A\&A, 505, 117

Chapman, S. 1917, MNRAS, 77, 540

Coelho, P. R. T. 2014, MNRAS, 440, 1027

Coelho, P., Barbuy, B., Meléndez, J., Schiavon, R. P., \& Castilho, B. V. 2005, A\&A, 443, 735

Dotter, A., Conroy, C., Cargile, P., \& Asplund, M. 2017, ApJ, 840, 99

Ernandes, H., Barbuy, B., Alves-Brito, A., et al. 2018, A\&A, 616, A18

Franchini, M., Morossi, C., Di Marcantonio, P., et al. 2018, ApJ, 862, 146

Fraunhofer, J. 1817, Denkschriften der Münch. Akademie der Wissenschaften, 5, 193

Fraunhofer, J. 1821, Denkschriften der Münch. Akademie der Wissenschaften, 8,1

Gao, X., Lind, K., Amarsi, A. M., et al. 2018, MNRAS, 481, 2666

Gratton, R. G., Bonifacio, P., Bragaglia, A., et al. 2001, A\&A, 369, 87

Gruyters, P., Korn, A. J., Richard, O., et al. 2013, A\&A, 555, A31

Gruyters, P., Lind, K., Richard, O., et al. 2016, A\&A, 589, A61

Husser, T.-O., Wende-von Berg, S., Dreizler, S., et al. 2013, A\&A, 553, A6

Husser, T.-O., Kamann, S., Dreizler, S., et al. 2016, A\&A, 588, A148

Kamann, S., Wisotzki, L., \& Roth, M. M. 2013, A\&A, 549, A71

Koleva, M., \& Vazdekis, A. 2012, A\&A, 538, A143

Koleva, M., Prugniel, P., Bouchard, A., \& Wu, Y. 2009, A\&A, 501, 1269

Korn, A. J., Grundahl, F., Richard, O., et al. 2007, ApJ, 671, 402

Lind, K., Korn, A. J., Barklem, P. S., \& Grundahl, F. 2008, A\&A, 490, 777

Liu, F., Asplund, M., Yong, D., et al. 2019, A\&A, 627, A117

Lovisi, L., Mucciarelli, A., Lanzoni, B., et al. 2012, ApJ, 754, 91

Martins, L. P., Coelho, P., Caproni, A., \& Vitoriano, R. 2014, MNRAS, 442, 1294

Martins, L. P., Lima-Dias, C., Coelho, P. R. T., \& Laganá, T. F. 2019, MNRAS, 484, 2388

Nordlander, T., Korn, A. J., Richard, O., \& Lind, K. 2012, ApJ, 753, 48

Prugniel, P., \& Soubiran, C. 2001, A\&A, 369, 1048

Prugniel, P., Vauglin, I., \& Koleva, M. 2011, A\&A, 531, A165

Richard, O., Michaud, G., Richer, J., et al. 2002, ApJ, 568, 979

Sánchez-Blázquez, P., Peletier, R. F., Jiménez-Vicente, J., et al. 2006, MNRAS, 371,703

Sharma, K., Prugniel, P., \& Singh, H. P. 2016, A\&A, 585, A64

Souto, D., Cunha, K., Smith, V. V., et al. 2018, ApJ, 857, 14

Stetson, P. B. 1987, PASP, 99, 191

VandenBerg, D. A., Richard, O., Michaud, G., \& Richer, J. 2002, ApJ, 571, 487 Wu, Y., Singh, H. P., Prugniel, P., Gupta, R., \& Koleva, M. 2011, A\&A, 525, A71 